\title{
BEHAVIORAL MODELS OF ISOLATED INDIVIDUALS AND ENTITIES
}

\author{
Anton MAŠTALSKÝ, Eduard DOLNÝ* \\ Training centre Lest, 96263 Pliešovce \\ *Corresponding author. E-mail: eduard.dolny@mil.sk
}

\begin{abstract}
The article describes the possibilities of using simulation in various fields, whether in aviation, education or in many other sectors. Modeling and simulation in computer training is nowadays used to increase civil security and is becoming particularly important for the development of today's society. Many simulator programs today allow the simulation of the influence of individual human factors and it is possible to involve them into the model situations, which for safety reasons is difficult to test in real life. However, accurate and effective models of human behavior also indicate great potential in military and crisis management. At present, these methods can be used to simulate the behavior of isolated individuals and entities that have a major impact, for example, on the spread of various viral diseases, depending on several factors. These factors include population, geographical location, age or education of the isolated and also social and cultural aspects, to which special space is devoted in this article. These factors can be used as inputs to the model for predicting the behavior of isolated groups of the population, on the basis of which it is possible to estimate in advance the spread of a possible epidemic, through various simulations.
\end{abstract}

Keywords: modeling and simulation; human behavior model; prediction model of human behavior

\section{INTRODUCTION}

Simulation is a general a term used to define any method of replicating real-world tasks, principally for education or research purposes. A simulation can be composed of computer-generated virtual scenes, situations or environments, actor-driven interactive role-play, or a simple instrument panel mock-up. For many in the aviation industry, the term simulation stirs thoughts of high-tech computer-generated replications that closely mimic the cockpit's appearance and function [1]. Simulations can be applied for a wide range of skill development in aviation. Historically, the simulator was dedicated to developing technical skills, such as stick and instrument procedures or rudder control [2]. In the last couple of decades, simulator training programs like Line Operators Flight Training (LOFT) [3] have extended the scope of training to include technical skills and coordination skills and team communication, such as in Crew Resource Management (CRM) [4]. The methods for providing aircrews with opportunities to practice CRM skills have been limited to roleplays and scenarios in operational flight trainers. There are disadvantages to both training methods; role plays have environmental lacks to unhelp crews act as they do in the cockpit, and simulator scenarios are limited by the cost and availability of the simulators [5]. Still, advances in simulation technology have allowed an impressive amount of training and instruction to be conducted on training simulators instead of existing systems. However, experiences with the acquisition and use of training simulators have not always been as successful, often lacking knowledge of didactic and training program development and inadequate simulator specifications [6].

Modelling and simulation in computer-assisted training for enhancing of the civil security becomes especially relevant to the development of today's society and the initiation of innovative technologies in everyday life. Current threats of terrorism, aviation attacks or hijack and also natural disasters need the coordination of many different structures. Therefore, simulation is a way to advance to check what the role should be run. The use of the latest technology allows recreating the objective reality and people's engagement in the newly designed virtual reality for training purposes. In contrast, global terrorism or aviation disasters models bring new challenges to the security of citizens and the recreation of virtual situations in the security context [7]. Human behaviour changes in reply to different situations are 
complex. Psychological changes are affected by various environmental, human and policy factors. Factors can be used as parameters to predict human behaviour change and can be helpful in understanding and expressing responses to quickly changing situations (e.g. emergencies) [8]. Predicting the collective behaviour of people during unusual explicit situations (e.g. evacuations of aircraft) is a significant difficulty involved with human behavioural factors such as fear, stress and panic. Many simulator programs present a multi-agent system for simulating the impact of individual human factors and can be applied to model situations that are hard to test in real life due to safety considerations [9]. A person who is a member of a group it behave differently from a person who perceives himself as an isolated individual. Group membership changes preferences over results, and the salience of the group influence the perception of the environment. Plus, group membership strongly affects individual decisions [10].

As human behaviour models play an essential part of living in situations, more social and psychological phenomena happen not as the outcome of isolated choices by people, but rather as the result of replicated interactions between multiple individuals over time [11]. This is also one reason why human behaviour and its simulation is complex in their structure. There are influenced by cognitive, emotional, physical, and social factors. Consequently, the human being is seen as a psychosomatic unit with cognitive capacities embedded in a social environment $[12,13]$. It is essential to control the behaviour of individuals not only in the course of management but also from other points of view. At present, some problems raised in practice (particularly in aviation) are concerned with inefficient behaviour control. Most traditional behaviour science research are conducted using the psychological method [14]. But precise and effective models of human behaviour suggest also great potential in military and crisis management applications [15]. However, developing high-fidelity models of human behaviour is challenging, usually resulting in high costs and long development times [16, 17]. Computer simulations of human behaviour increased rapidly during the 1950s - 1960s, but moderately during the 1970s. Although it has limitations and advantages, it is the best methodology for training and particular tasks [18]. Simulations also play an important role in resolving crisis situations associated with the isolation of humans, for example, in the spread of various infectious diseases, which can be predicted accordingly.

\section{ISOLATION AND ITS IMPACT}

There are cases, where there may be circumstances in which certain sections of the population are placed in temporary or permanent isolation from other people. The introduction of the isolation of risk groups of the population is one of the solutions used in the event of a pandemic. Isolation or isolation is a general term for separating an individual or entity from other groups of the population. Sociology uses the concept of social isolation, in which there is an absence of contact with people to whom the individual attaches social importance. From a biological point of view and in medicine, when the term separation or quarantine is also used, this is the so-called the physical isolation of an individual or group of the population that causes an infectious disease.

As it can be seen from Figure 1, the role of such isolation is primarily to protect the population from the spread of infectious disease [19, 20,21].

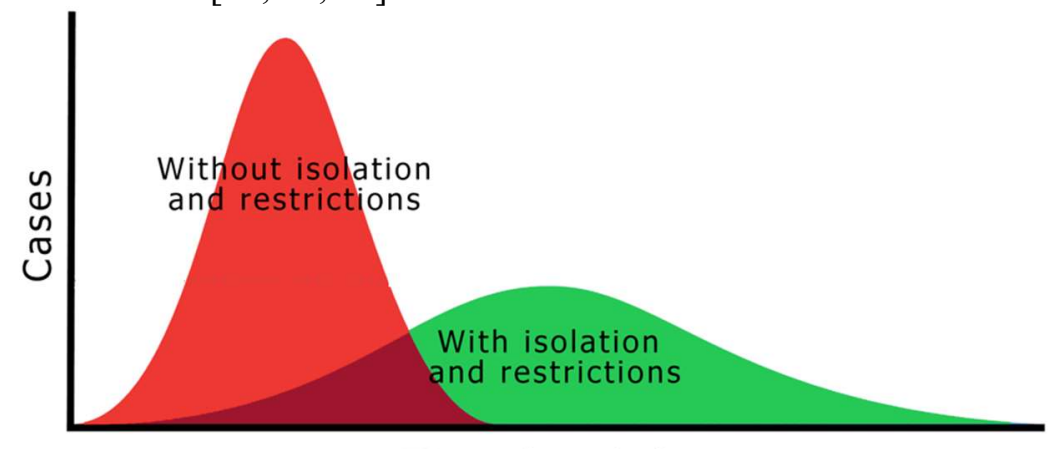

Time (weeks)

Figure 1 Spread of various viruses before and after the introduction of isolation and restrictions 
Physical isolation or quarantine is crucial to slow the spread of infectious diseases. Such social isolation can affect the mental health of individuals. As mental and physical health are interlinked, the adverse health consequences of isolation are manifested, for example, by insomnia or impaired immune function, cardiovascular problems or cognitive impairment. Loneliness is also associated with depression or anxiety [22] and, last but not least, with a longer period of such separation, this will result in a breach of the measures.

\section{MODELS OF HUMAN BAHAVIOR}

Human behavior has a significant impact on the spread of viral diseases, which is generally global, and the highest incidence of these diseases is in the autumn and winter months. Many viral diseases are known for their high infectivity and are common in the population. The source of the infection are humans and the spread takes place mostly in collectives. The virus is most often transmitted from person to person by drip infection. Preventive measures against dissemination are, in particular, In compliance with personal hygiene, environmental hygiene, whether in the household or in collective facilities of the school, medical or social type.

Other aspects of human behavior, such as the spread of viral diseases, also depend on other aspects, including population, age, geographical location, high-concentration areas and, of course, population culture - see Figure 2. Adequate models, then allow modeling of the impact of different situations, which can help in the implementation of measures within the group of people or the area in which they live [23].

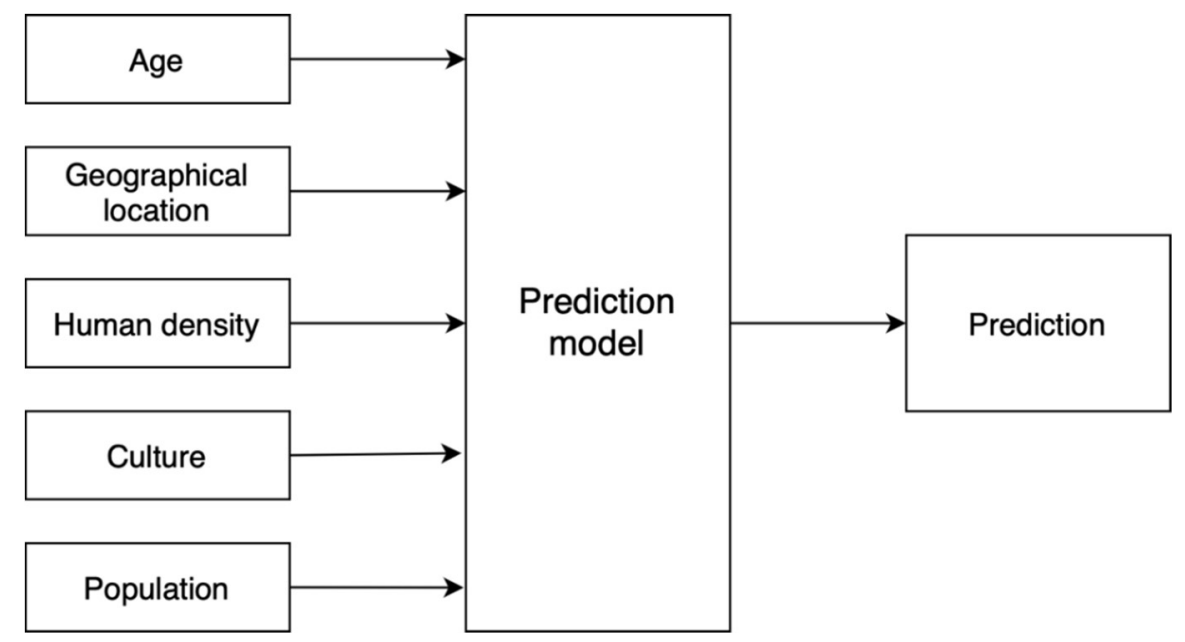

Figure 2 Prediction model with defined inputs

In order to limit the spread of the viral disease, various types of measures and restrictions are imposed on the public movement of the population. The introduction of social distance as one of the measures against the spread of viral disease can have detrimental effects on physical as well as mental well-being in various sections of society and age groups. In global pandemics, proliferation control depends on several factors $[24,25,26]$.

\subsection{Age factor}

One of the factors is the age of the population when one of the studies took a sample of people aged 18 to 85 and developed a comprehensive analysis of the socio-demographic and psychosocial effects of age on the perception of disease risks as well as safe compliance. The results show that the risk perception and safe behavior decrease with age. It is also pointed out that anxiety, optimism and social isolation affect the perception of the risks associated with the disease at different ages of the population, and a group of middle-aged and younger people perceive these risks [24, 25, 26]. 
Safe behavior and risk perception in different age groups also result in the spread of the disease. In areas with a younger population, the possibility of the virus spreading is greater as the elderly population tends to make more social contacts. From several studies, we can state that children and adults under the age of 65 account for the highest rate of infected cases. The total number of infected, then depends on the reproductive number, the rate and the rate of infection, the rate of progression of the infection, the rate of recovery of the infected, the rate of mortality of the infected, vaccination and treatment of the infected $[27,28,29]$.

\subsection{Geographical location factor and population density factor}

Geographical location and population density factor also play important role. Therefore, it is necessary to consider a strategy where it is necessary to minimize the level of contact with infected individuals and thus limit access to places with a large concentration of population. These places include social venues, pubs, bars, restaurants, shopping malls, cinemas and fitness centers [24]. Also, the rate of spread of the infection also depends on the geographical location, which is directly related to the population itself. In general, the spread of various viral diseases is faster in cities than in rural areas. This is mainly due to population density and social interaction in these geographical areas. Historically, various pandemics have tended to affect large cities more than rural areas. Large cities also transport hubs for international transport networks as well as surrounding regions. On this basis, there is a suggestive evidence that such interconnected areas contribute to the spread of various diseases. These impacts can be mitigated by the isolation of individual groups of the population, which is also directly related to the geographical location. The rural environment is characterized by greater physical isolation due to lower population density, long distances to other cities, as well as a lack of transport and infrastructure. This contributes to the isolation of the population, but at the same time it can also contribute to the feeling of loneliness, of people in these regions, in contrast to large urban areas, which can result in future mental health problems [30, 31].

\subsection{Cultural factor}

Culture is one of the most costly ways of shaping people's behavior. Culture also affects the spread of viral disease. Responses to the introduction of social isolation measures vary from one culture to another. These reactions will vary depending on the structure of the family background, the way of life and the economic situation. The effect on propagation can then be greater or less. It has been shown that there is a higher rate of spread of the virus between ethnic groups and immigrants in different populations [32]. The impact of cultural behaviors such as handshakes or face kisses, which are widely accepted greetings internationally, can contribute to the spread of viruses and bacteria. The complexity and diversity of cultural practices is another factor that is important to consider. Among the cultural studies, they say that each culture has its own specific beliefs related to health and disease perceptions. The impact of culture on people's behavior in isolation is not negligible and must be seen as one of the inputs in the behavior model [33].

\section{CONCLUSIONS}

Simulation methods are currently widely used in various industries, through aviation, education, in modeling the behavior of people in crisis situations and in many other industries. However, modeling and simulation are also currently used to address issues related to the spread of viral diseases and the isolation of humans. Isolation of humans has a direct impact on the spread of various infectious diseases. Reducing social contacts is one of the most effective tools in combating the spread of such diseases. At the same time, however, such long-term isolation can also have the opposite effect, mainly due to the fact that long-term isolation of people adversely affects their mental health, which tends to violate isolation and various restrictions. However, it all depends on the various factors that were described in the article, whether it is the age of the population, population density, geographical location and other factors. It is these factors that can be the input parameters in the further model of human behavior in 
further research, on the basis of which it would be possible to simulate various situations in the spread of viral diseases.

However, the prediction in the behavioral models is still a challenging task because of the so many input factors which is in many cases difficult to quantify. Therefore, the involving of the prediction into these models is usually based only on the conventional statistical approaches and will be a part of the further research.

\section{Acknowledgement}

This work was supported by the project of Research Agency entitled Mobile Monitoring System for the Protection of Isolated and Vulnerable Population Groups against Spread of Viral Diseases, ITMS2014+ code 313011AUP1, co-funded by the European Regional Development Fund under the Operational Programme Integrated Infrastructure.

\section{References}

[1] Entsch, F., Curtis, M. Simulation in Aviation Training. Routledge. 2017

[2] Williges, B. H., Roscoe, S. N., Williges, R. C. Synthetic Flight Training Revisited. Human Factors. 1973. p. 543-560.

[3] Butler, R. E. LOFT: Full-mission simulation as Crew Resource Management Training. 1993.

[4] Helmreich, R. L., Merritt, A. C., Wilhelm, J. A. The evolution of crew resource management training in commercial aviation. The International Journal of Aviation Psychology. 1999. p. 1932.

[5] Baker, D. \& et al. Aviation Computer Games for Crew Resource Management Training. The International Journal of Aviation Psychology. 1993. p. 143-156.

[6] Lee, A. T. Flight simulation: virtual environments in aviation. Routledge. 2017.

[7] Goranov, Z. Computer-assisted Courses. In: Proceedings of International Conference on Application of Information and Communication Technology and Statistics in Economy and Education (ICAICTSEE). International Conference on Application of Information and Communication Technology and Statistics and Economy and Education (ICAICTSEE). 2013.

[8] Cheng, Y. \& et al. Human behavior under emergency and its simulation modeling: a review. In: International Conference on Applied Human Factors and Ergonomics. Springer. Cham, 2018. p. 313-325.

[9] Sharma, S., Singh, H., Prakash, A. Multi-agent Modeling and Simulation of Human Behavior in Aircraft Evacuations. IEEE Transactions on aerospace and electronic systems. 2008. p. 14771488.

[10] Charness, G., Luca R., Aldo R. Individual Behavior and Group Membership. American Economic Review. 97. 2007. p. 1340-1352.

[11] Smith, E. R., Conrey, F. R. Agent-based modeling: A new approach for theory building in social psychology. Personality and social psychology review. 2007. p. 87-104.

[12] Botvinick, M. M. Hierarchical models of behavior and prefrontal function. Trends in cognitive sciences. 2008. p. 201-208.

[13] Scmidt, B. The modelling of human behaviour: The PECS reference models. Delft: SCS-Europe BVBA. 2000.

[14] Sun, SR., Meng, P. Behavior element, behavior state and behavior model. In: Proceedings Of The 2001 International Conference On Management Science And Engineering. Harbin. China. p. 2381-2385. 2001.

[15] Van Doesburg, WA.: Quality assessment of human behavior models. Procedings Of The 11th Iasted International Conference On Artificial Intelligence And Soft Computing, Palma de Mallorca, Spain. 2007. pp. 137-144. 2007. 
[16] Taylor, G., Wray, R. E. Behavior design patterns: Engineering human behavior models. In: Proceedings of the Behavior Representation in Modeling and Simulation Conference. 2004.

[17] Yordanova, K. Human behaviour modelling approach for intention recognition in ambient assisted living. In: Ambient Intelligence-Software and Applications. Springer. Berlin. Heidelberg. 2011. p. 247-251.

[18] Starbuck, W. H. Computer simulation of human behavior. Behavioral Science. 1983. p. 154165.

[19] Rudan I. Health effects of human population isolation and admixture. Croat Med J. 2006. p. 526-531. Available at: https://www.ncbi.nlm.nih.gov/pmc/articles/PMC2080449/

[20] Postrehy behaviorálnych vied $\mathrm{k}$ pandémii COVID-19. Available at: https://practicalhealthpsychology.com/sk/2020/04/insights-from-behavioural-science-for-thecovid-19-pandemic/

[21] Brooks, S. K., et al. The psychological impact of quarantine and how to reduce it: rapid review of the evidence. The Lancet. 2020. DOI: https://doi.org/10.1016/S0140-6736(20)30460-8

[22] Understanding the Effect of Social Isolation on Mental Health. Tulane University. 2020. Available at: https://publichealth.tulane.edu/blog/effects-of-social-isolation-on-mental-health/

[23] Vyklyuk, Y., Manylich, M., Škoda, M., Radovanović, M.M., Petrović, M.D. Modeling and analysis of different scenarios for the spread of COVID-19 by using the modified multi-agent systems - Evidence from the selected countries. Results Phys. 2021. DOI: 10.1016/j.rinp.2020.103662.

[24] Banerjee, D., Rai, M. Social isolation in Covid-19: The impact of loneliness. International Journal of Social Psychiatry. 2020. DOI:10.1177/0020764020922269

[25] Pasion, R., Paiva, T. O., Fernandes, C., Barbosa, F. The AGE Effect on Protective Behaviors During the COVID-19 Outbreak: Sociodemographic, Perceptions and Psychological Accounts. Laboratory of Neuropsychophysiology. University of Porto. Porto. 2020.

[26] Oster, A. M., et al. Transmission Dynamics by Age Group in COVID-19 Hotspot Counties. Morbidity and Mortality Weekly Report. United States. 2020. Available at: https://www.cdc.gov/mmwr/volumes/69/wr/pdfs/mm6941e1-H.pdf

[27] James, A., et al. Model-free estimation of COVID-19 transmission dynamics from a complete outbreak. 2021. DOI: https://doi.org/10.1371/journal.pone.0238800

[28] Rahman, A., Kuddus, M. A. Modelling the Transmission Dynamics of COVID-19 in Six HighBurden Countries. BioMed Research International. 2021.

[29] Davies, N.G., et al. Age-dependent effects in the transmission and control of COVID-19 epidemics. Nat Med. 2020. p.1205-1211. DOI: https://doi.org/10.1038/s41591-020-0962-9

[30] Herron, R.V., et al. Conversations in Times of Isolation: Exploring Rural-Dwelling Older Adults' Experiences of Isolation and Loneliness during the COVID-19 Pandemic in Manitoba, Canada. Int J Environ Res Public Health. 2021. DOI:10.3390/ijerph18063028

[31] Matheson, J., et al. Why has coronavirus affected cities more than rural areas? Available at: https://www.economicsobservatory.com/why-has-coronavirus-affected-cities-more-rural-areas

[32] How culture affects the spread of pandemic like COVID-19. Available at: https://www.futurity.org/covid-19-culture-history-2318752/

[33] Bruns, D. P., et al. Facts, Cultural Considerations, and Risk of Stigmatization. Journal of Transcultural Nursing. 2020. DOI: https://doi.org/10.1177/1043659620917724

Received 11, 2021, accepted 12, 2021

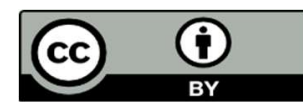

Article is licensed under a Creative Commons Attribution 4.0 International License 